\title{
Welcome to Therapeutic Delivery
}
"This exciting new journal will cover a plethora of new delivery technologies and applications with a fresh, interdisciplinary approach, via original cutting-edge research papers and critical reviews from diverse fields, with a particular focus on translational outputs for the effective treatment of diseases."

\begin{abstract}
Welcome to Therapeutic Delivery
It is my pleasure to welcome you to the inaugural issue of Therapeutic Delivery. This exciting new journal will cover a plethora of new delivery technologies and applications with a fresh, interdisciplinary approach, via original cutting-edge research papers and critical reviews from diverse fields, with a particular focus on translational outputs for the effective treatment of diseases.
\end{abstract}

\section{Journal content}

Therapeutic Delivery will go a long way towards improving our understanding of new delivery technologies by bringing cutting-edge research from across the world into one common platform. Basic research is the foundation of future invention and commercialization of delivery technologies, so we invite the submission of both basic mechanistic, applied, clinical and translational research papers to complement the core content of critical reviews.

Patents are a vital source of information on new delivery technologies, so reviews of relevant intellectual property literature will be provided. We will keep our readers up-to-date with reports from the latest conferences, book reviews and interviews with leading figures from academia and industry. Timely editorial articles will provide a forum for debate on issues of topical importance pertaining to the scientific, regulatory and societal aspects of delivery of therapeutics to patients.

\section{Industrial \& regulatory perspectives}

Therapeutic Delivery will provide an industrial perspective on delivery systems, the importance of which cannot be overemphasized. When the recently marketed insulin inhalation device was withdrawn from sale, the repercussions extended to academic researchers in the field as well as to other companies that were in late-stage clinical trials with similar products. Nevertheless, inhalation for local and, to a lesser extent, systemic delivery of therapeutics remains one of the more promising routes for delivery of macromolecules. It is a only a matter of time before interest reemerges in this technology. In fact, Phase III clinical trials of inhaled insulin have recently been successfully completed by another company and product approval in the near future is quite possible. It is important for research scientists to learn from setbacks and keep working to advance the science that will later benefit patients by new product approvals or, more indirectly, by discovering new applications or expanding our collective knowledge.

\section{"Therapeutic Delivery will provide an industrial perspective on delivery systems, the importance of which cannot be overemphasized."}

Many new biopharmaceuticals face significant delivery challenges to the extent that they will never reach the marketplace if innovative delivery technologies and strategies are not invented. A timely discussion of such emerging technologies will be provided in Therapeutic Delivery.

The choice of an appropriate drug molecule for a particular delivery system to satisfy an unmet therapeutic need will make US FDA approval more likely and also establish the delivery technology platform enabling the launch of future drug molecules with an easier regulatory pathway. Indeed, consideration of regulatory guidelines is of vital importance to the success of any therapeutic. Therapeutic Delivery will provide the reader with an appreciation of the intricacies of combining new technologies with unmet medical needs, commercial differentiation and meeting regulatory requirements.

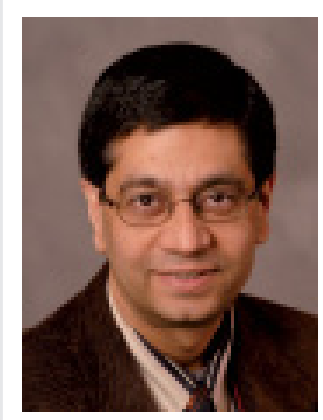

Ajay K Banga

Department of Pharmaceutical Sciences, Mercer University, 300I Mercer University Drive, Atlanta, GA 3034I, USA E-mail: banga_ak@mercer.edu 


\section{Themed issues}

To ensure that sufficient focus is given to the key research areas, Therapeutic Delivery will regularly publish themed issues. Therapeutic Delivery will take a flexible approach to these themed issues and, by remaining flexible and responding to 'hot' areas of research, will provide timely articles focusing on important advances relating to:

- Delivery in specific therapeutic areas (e.g., oncology and cardiology)

- Delivery of particular drug molecules (e.g., small molecules and biopharmaceuticals)

- Delivery routes (e.g., parenteral, oral, inhalation, mucosal and transdermal)

- Targeting at the molecular, subcellular, cellular, organ and systemic levels

- PEGylation and other chemical conjugation technologies

- Nanotechnology, liposomes and microspheres

- Innovative delivery devices and systems (e.g., injector pens, pumps and inhalers)

\section{A diverse research field}

Today's successful therapeutic delivery strategies rely upon highly interdisciplinary research and collaboration across an array of research areas. I would like to take an example of a specific delivery route to highlight the importance of interdisciplinary research and to discuss the intricacies that are an inevitable part of any delivery strategy.

As my research over the years has focused on transdermal delivery it was an obvious choice, but the principles are equally as applicable to other delivery routes. Transdermal delivery has been quite a success story with over 15 different drug molecules on the market, despite the fact that delivery is predominantly limited to moderately lipophilic drug molecules. Several nontraditional technologies are currently being investigated to expand the scope of transdermal delivery and to enable the delivery of hydrophilic drugs, both small molecules and biopharmaceuticals.

One of the many exciting developments in this field has been the recent interest in microporation approaches that create microscopic holes (microchannels) in the skin to enable the delivery of hydrophilic molecules and macromolecules, which would otherwise not be able to cross the lipophilic barrier of the upper layer of the skin (stratum corneum). The microchannels created by this minimally invasive technology are much smaller than the holes made by hypodermic needles. Much interest has centered on microneedles, which are typically made of metal, polymers or even sugar and combine the effectiveness of needle-based delivery with the patient acceptance of patch technology. They are typically $100-750-\mu \mathrm{m}$ long, with a narrow radius of curvature at the tip, allowing easy penetration into the skin. The use of microneedles coated with vaccines is also gaining considerable interest and could see potential use for worldwide mass immunization programs. Other technologies such as thermal, radiofrequency or laser ablation can also create microchannels in the skin, and several companies are actively pursuing these microporation technologies. Resolution of potential hurdles to commercialization relating to availability of cost-effective scale-up technologies, bioavailability considerations and a better understanding of pore closure kinetics and immunogenicity considerations are required and offer opportunities for further research in these areas. Other promising transdermal technologies that have already seen some commercial success include the use of electric current (iontophoresis) or ultrasound (phonophoresis) to enhance delivery of drug molecules into and through the skin. Some setbacks with marketed iontophoresis products have been a learning experience and present opportunities for further research.

\section{"Many new biopharmaceuticals face significant delivery challenges to the extent that they will never reach the marketplace if innovative delivery technologies and strategies are not invented. A timely discussion of such emerging technologies will be provided in Therapeutic Delivery."}

Other transdermal technologies under investigation to enable systemic drug delivery include electroporation, microdermabrasion, controlled heat-assisted delivery, particlemediated delivery and chemical enhancers. Each of these technologies has its own advantages and limitations. Iontophoresis is typically considered to enable delivery of (preferably charged) hydrophilic macromolecules up to a size of approximately $12 \mathrm{kDa}$. On the other hand, microporation technologies have 
no such size limit for drug molecules and can deliver all the way up to monoclonal antibodies $(\sim 150 \mathrm{kDa})$ and even DNA or small particulates. However, currently only small quantities (a few milligrams) can typically be delivered by any of these technologies. Nanotechnology or other approaches may allow delivery of larger doses and more research is needed in this direction. Some of these technologies can enable the development of all-inclusive wearable patches while others may need a handheld activator for the patch. Advances in fields such as chemistry, biology, electrical and biomedical engineering, materials science, medicine and pharmaceutical sciences, and fruitful collaborations among these scientists, have been instrumental in the development of these transdermal delivery technologies.

Similarly, other delivery routes and technologies have their own intricacies and complexities that all benefit from interdisciplinary research. Therapeutic Delivery recognizes the importance of this diverse research platform and focuses on how the technological, pharmacological, clinical and physiological aspects come together to successfully deliver modern therapeutics in a way that is right for patients.

\section{Benefits of Therapeutic Delivery}

There are many advantages to publishing your work in Therapeutic Delivery, including high visibility and impact, freedom from author charges, rapid publication in both print and online formats (typically $8-10$ weeks from submission to acceptance) and speedy, rigorous peer review.

We hope that readers will enjoy the journal's industry-leading engaging layout. Articles published in Therapeutic Delivery include enhanced features to ensure that busy researchers have rapid access to practical information: an executive summary cuts directly to the major points in the article; key terminology is highlighted in the text and explained; authors are challenged to look at the future potential of their work by including a 'Future perspective' section in their review and research articles; and the most important references are highlighted with author commentary.

I am excited about the launch of Therapeutic Delivery and wish the very best to the researchers who will disseminate their findings through the pages of this journal in the years to come, and successfully bring many of these delivery systems from the bench to the bedside.

\section{Financial \& competing interests disclosure}

The author has served as a consultant or has been funded by companies that support some of the technologies listed in this article. The author has no other relevant affliations or financial involvement with any organization or entity with a financial interest in or financial conflict with the subject matter or materials discussed in the manuscript apart from those disclosed.

No writing assistance was utilized in the production of this manuscript. 